\title{
The relationship between apoptosis and aging
}

\author{
Bin Lu ${ }^{1,2}$, Hong-Duo Chen ${ }^{1}$, Hong-Guang $\mathrm{Lu}^{2 *}$ \\ ${ }^{1}$ Department of Dermatology, Affiliated Hospital of China Medical University, Shenyang, China \\ ${ }^{2}$ Department of Dermatology, Affiliated Hospital of Guiyang Medical University, Guiyang, China \\ Email: hongguanglu@hotmail.com
}

Received 15 August 2012; revised 20 September 2012; accepted 29 September 2012

\begin{abstract}
Apoptosis is involved in aging and age-related disease, with respect to aging, apoptosis acting in a cell typespecific manner. The rate of apoptosis is elevated inmost types of aging cell populations and organs. In stable cells and certain continuously dividing cells, apoptosis serves to eliminate presumably dysfunctional cells that show homeostatic failure due to oxidative stress, glycation, and DNA damage, thereby maintaining homeostasis in the body. What's more, apoptosis, at least in part, plays some important role inthe regulation of aging process and anti-tumorigenesis in mammals. Age-enhanced apoptosis may be aninnate protective mechanism against age-associated tumorigenesis. There is clear evidence to indicate that senescent cells are remarkably resistant to apoptosis. The intensity of global apoptosis and autophagy clearance significantly declines in humans during aging, as aging repress the apoptotic response. In this paper, we will review the relationship between apoptosis and aging, and outline how are they interact each other.
\end{abstract}

Keywords: Apoptosis; Aging; Senescence

\section{INTRODUCTION}

Apoptosis is a process whereby cells activate an intrinsic cell suicide program that is one of the potential cellular responses, such as differentiation and proliferation. It has been defined in 1972 by Kerr et al. in contrast to necrosis, which is a cell death generally due to aggressions from the external Medium [1]. Many theories of apoptosis have been proposed, and they can be divided into intrinsic and extrinsic pathways. One of most important extrinsic pathways is radiation-induced apoptosis, that is considered to be one of the main cell death mechanisms following exposure to irradiation [2], especially ultraviolet radiation b (UVB) [3]. The intrinsic pathways including many hypothesis, and many factors involved in them,

"Corresponding author. such as gene [4,5], DNA damage [6], some proteins [7, 8], and kinases or phosphatases [9,10], lysosomes [11], mitochondria [12], persistent stress [13], and ROS [14]. Apoptosis is a vital component of the evolutionarily conserved host defense system of organisms. When subjected to pathological attack, apoptosis is a guardian of tissues, since it can cleanse of unfit and injured cells without evoking inflammation [15]. In persistent stress, such as in aging, increased apoptotic resistance can lead to the survival of unfit cells that are not able to maintainproper housekeeping functions. This increase in apoptotic resistance may be relevant if one considers the context of tissue integrity during aging, but it takes place at the cost of housekeeping potential and leads to a sensecent phenotype in post-mitotic cells.

Aging is an essential, inevitable physiological phenomenon characterized by a progressive accumulation of deleterious molecular damages in cells and tissues during the post-maturational deterioration, which decreases the ability to survive and increases risk of death [16]. The aging process has many facets and multiple causes. The primary molecular phenotype of aging is the stochastic occurrence and accumulation of molecular damage leading to a progressive increase in molecular heterogeneity and functional impairment [17]. Cellular senescence is the state where cells have irreversibly lost their proliferation ability, and they exhibit deficiencies in maintaining their homeostatic processes [18]. The number of sensecent cells increases in tissues with aging. Many theories about the causes of aging have been proposed [19], and could be divided into two broad categories: the stochastic theories and the developmental-genetic theories [20]. Age-related degeneration can be a consequence of a genetic program or it may be an entropic process [21]. Ultimately, disorders in housekeeping ability jeopardize homeostasis and expose cells to apoptotic forms of cell death.

Accumulating evidence strongly suggests that deregulation of apoptosis is associated with the aging process [22], however, it is still debatable whether aging suppresses or enhances apoptosis in vivo. At present, it is unclear whether the alterations in apoptosis observed 
during ageing are consequences of ageing or participate in the normal ageing process. In this paper, we will review apoptosis application in ageing, and discuss what role of apoptosis involved in aging process.

\section{APOPTPSOS IS INVOLVED IN AGING AND STRONG ASSOSIATIED WITH AGE-RELATED DISEASES}

It has been elucidated that the possible role of apoptosis in aging and age-related diseases [23]. Apoptosis, at least in part, plays an important role in the aging process and age-associated tumorigenesis in mammals [24]. The rate of apoptosis is elevated inmost types of aging cell populations and organs, such as brain, cardio-vascular system, immune system, endocrine system, intestine, reproducetive system and eye [25]. Apoptosis increased in these cells or organs is regarded as a protective mechanism of the organism against an accumulation and spread of defective cells, but the preponderance of this dismantling system seems to highlight the age-associated decline and deterioration in tissue and organ structure and function [25]. Age-associated changes of the genes involved inapoptosis could be among the most prominent alterations of gene expression in aging. On the one hand, lots of genes promote apoptosis decrease in aging, such as the gene of p53 and caspase family genes including Casp3, Casp8, and Casp9 [26-29]. On the other hand, the expression of anti-apoptotic genes such as Bcl-2 was upregulated in aging [26]. P53 functions as a longevity assurance gene and a regulator of aging [3]. In several mouse models, persistent low-level activation of p53, either through deregulated expression of p53 itself or in response to constitutive stress like DNA damage leads to premature aging [30,31]. Members of the Bcl-2 family proteins are critical death regulators that reside upstream of antiapoptotic [32]. Either promoting apoptosis genes decrease or anti-apoptotic genes decrease in aging process, it can lead to aging in the end. So we deduce that relationship of apoptosis and aging process is as following: 1) Apoptosis is increased in most of organs with aging process; 2) Aging is able to restrain apoptosis to appropriate states through genes or their products; 3 ) Aging process is the result of the interaction of the apoptosis and aging.

Changes in apoptosis have path-physiological conesquences in aging as a sentinel homeostatic pathway, much apoptosis can yield tissue degeneration [33], while little apoptosis allows either dysfunctional cells to accumulate or differentiated immune cells topersist [34], and inappropriate apoptosis may contribute to age-related diseases and cancer [35]. It has been suggested that aging related degenerative neurological diseases, such as Alzheimer disease [36] and Parkinson's disease [37], and that apoptosis is one of the underlying pathogenic mechanisms of these neurological diseases [38]. Amyotrophic Lateral Sclerosis (ALS) is an adult onset fatal disease. The feature of this disease is the degeneration of motor neurons in the spinal cord and brain. Apoptosis may play a role in this disease [39]. Huntington's disease (HD) is a progressive, fatal neurological condition, and cells with mutant Huntington proteins may be more sensitive to apoptosis induced by ageing associated oxidative stress [40]. Other post mitotic tissues, like skeletal muscle and heart, also exhibit age-related apoptotic changes [41,42].

\section{APOPTOSIS BENEFIT THE ORGANISM AND PREVENTING TUMORGENESIS}

It has been suggested that reprogramming cells to apoptosis may be a means to reduce cancer and eliminate one cause of aging. An organism is constantly exposed to a variety of stressful conditions. It would be disastrous for the organism if it could not prevent the initiation of apoptosis when the initiation signal is due to reparable or minor damage. An important role of apoptosis is to ensure the efficient removal of the cells whose continuing existence is detrimental to the organism [43]. Apoptotic destruction of a "unwanted" cell makes it ready for being adhered and absorbed by a phagocyte [44]. The decline in apoptotic response during aging could lead to loss of phenotypic fidelity and a subsequent accumulation of cells with macromolecular damage, including damage to DNA [45]. Apoptosis protects against the accumulation of pre-cancerous mutations by eliminating cells harboring excessive DNA damage [46]. Because non-senescent old cells are still dividing, their apoptosis deficiency will result in DNA replication past DNA lesions [47]. Apoptosis is also essential to wound repair [48]. Wound repair is impaired with aging [49]. During wound maturation, fibroblasts need to be eliminated to reduce the production of collagen and concomitant vascularity [48]. It is noted that apoptotic fibroblasts are less abundant in the dermal granulation tissue of older rats than in younger rats [50]. It is possible that delayed skin wound repair of older individuals is due, at least in part, to an apoptosis defect in older fibroblasts [47].

Ageing has been described as one of the most potentcarcinogens [51]. It is proposed that the sharp rise in cancer incidence with age may be partly related to a systemic failure of apoptosis [52,53]. Failure of cell death programs at old age would be especially harmful for tissues with retaining proliferative ability and could explain to some extent the well-documented increased incidence of cancer and accumulation of DNA damage with age [51]. Polyak et al. [54] observed a reduced apoptotic 
response of lymphocytes to 5 Gy radiation in mice as they aged, and Suh et al. [53] found a reduced apoptotic response in rat liver cells exposed to methyl methane-sulphonate. Both of them discuss the possibility that the sharp rise in cancer incidence with age may be partly related to a systemic failure of apoptosis [52]. The dates from mice also support the views that apoptosis are a defense against cancer [30]. It is thought that apoptosis may be protective against cancer in some tissues, and the decrease in apoptosis with age because of less efficient cellular signaling and regulation may promote cancer ogenesis [55]. p53 is a potent initiator of both apoptosis and cell cycle arrest, and the decline in p53 response to stress at older ages in animals could contribute to the observed dramatic increases in cancer incidence, and provides a reasonable explanation for the correlation between tumorigenesis and aging [56]. The decline in p53 at older ages could contribute to the observed dramatic increases in cancer incidence [56]. It is certainly possible that some of the mutations which occur early in tumor formation may increase the mutation rate and lower the time to tumor formation and counter acting this process is the p53-mediated elimination of clones of cells via apoptosis that contain these mistakes [55]. Moreover, numerous studies have indicated that anticancer therapies primarily act by activating the apoptosis response pathway in tumor cells [57-61].

\section{CELLULAR SENESCENCERESIST TO APOPTOSIS}

Cellular senescence is the state where cells have ireversibly lost their proliferation ability, and they exhibit deficiencies in maintaining their homeostatic processes $[18,62]$. Senescent cells display a number of distinctive characteristics, such as increased ROS production, increased oxidative damage, increased glycation damage, reduced heat shock protein expression, accumulation of defective proteins together with decline in apoptotic protein function [63]. If cells are unable to repair DNA damage, apoptosis may ensue, followed by replacement via division of another cell. Senescent cells are resistant to apoptosis and more sensitive to cell injury [55], and increased resistance toapoptosis is a significant functional hallmark of senescent cells. It is demonstrated that replicative senescent human fibroblasts were resistant to apoptotic insults. Moreover, fibroblasts of progeroidWerner syndrome patients exhibit attenuation of p53induced apoptosis [64], and p53-mediated gene expression is involved in the appearance of apoptosis resistance in human skin fibroblasts [3]. Recently, it is reported that the appearance of resistance to UVB-induced apoptosis clearly precedes the progressive development of replicative senescence inhuman fibroblasts [47]. Resistance to apoptosis might partly explain why senescent cells are so stable in culture, and it attribute might also explain why the number of senescent cells increases with age [65]. However, it is still largely unclear whether age-related cellular senescence and quiescence of mitotic cells can elicit resistance to apoptosis in tissues, comparable to apoptotic resistance in cultured cells. The problem seems to be that apoptotic cells are not frequently encountered in tissue sections, this probably being attributable to either their fast clearance or to apoptotic resistance under normal conditions.

The mechanisms by which senescent cells resist apoptosis are poorly understood. In some cells, resistance might be due to expression changes in proteins that inhibit, promote or implement apoptotic cell death [66]. In others, p53 might preferentially transactivate genes that arrest proliferation, rather than those that facilitate apoptosis [67]. However, there are clear cell type-dependent differences in the apoptotic resistance induced by growth arrest and cellular senescence in cultured cells. For example, senescent keratinocytes are resistant to UV-induced apoptosis, as are skin fibroblasts [68], but senescent endothelial cells are more vulnerable to apoptosis if examined under in vitro cell culture conditions [69].On the other hand, endothelial cells incultured arteries have been reported to display an increased resistance to apoptosis in long-lived rodents [70]. One explanation could be that senescent endothelial cells in culture undergo anoikis, not apoptosis. Anoikis is a specific apoptotic process triggered by the loss of extracellular matrix interactions [71]. The mechanisms conferring resistance to anoikis are quite different from those inhibiting apoptosis.

\section{ORGANISMAL AGING REPRESS APOPTOSIS AND AUTOPHAGIC CLEARENCE}

As mentioned above, senescence in cultured cells resistant to apoptosis, however, it is not clear whether tissues or organizes can elicit resistance to apoptosis. The problem seems to be that apoptotic cells are not frequently encountered in tissues, this probably being attributable to either their fast clearance or to apoptotic resistance under normal conditions. Recently, it is demonstrated that the intensity of global apoptosis significantly declines in humans during aging [72]. With increasing age there was a decrease in apoptotic markers and pro-apoptotic factors and an increase in anti-apoptotic factors in circulation [72]. Moreover, several studies have demonstrated that aging decreases the apoptotic response to genotoxic stress [53,73]. Apoptosis is also involved in the age-related remodeling of the immune system, i.e., decreased proliferation capacity and increased resistance to apop- 
tosis [74]. De Martinis et al. [75] have described the cells of the immune system undergo two different kinds of apoptotic processes: activation-induced cell death (AICD), geared towards the elimination of unnecessary lymphocytes following clonal expansion, and damage-induced cell death (DICD), particularly important for preventing the onset of neoplastic proliferations. Organismal aging seems to potentiate the apoptotic pathway of AICD and decreases the sensitivity to DICD [75]. The apoptotic deficiency in immuneosenescent lymphocytes can also provoke autoimmune responses in the elderly. Lymphocyte senescence is probably one form of the rather common senescence-associated secretory phenoltype, and in that way it supports the appearance of age-related proinflammatory status, which accelerates the aging process and aggravates age-related degenerative diseases.

However, increased resistance to apoptosis of sensecent cells represents a threat to functional integrity, such as the immune system, skin, and vascular endothelium. Increased tolerance of cells to molecular damage leads to the accumulation of intracellular waste products within nondividing cells, e.g., senescent cells and post-mitotic cells. This activates autophagocytosis, a major cellular housekeeping mechanism that can remove damaged molecules and organelles. However, many studies have demonstrated that autophagy clearance declines during the normal aging process [76,77]. Recent studies have revealed that autophagy has an effect or role in the induction of the senescent phenotype [78-80] It is demonstrated that autophagy is clearly activated during the transition phase into cellular senescence in fibroblasts $[79,80]$. The induction of autophagy in the early phase of the accelerated aging process seems to be a survival mechanism that compromises the metabolic disorders in the transition phase in order to inhibit apoptotic cell death [81].

\section{CONCLUSION AND PERSPECTIVES}

Apoptosis is involved in aging and age-related disease, however, apoptosis serves to eliminate presumably dysfunctional cells and protect organism from cancer. On the other hand, senescent cells are remarkably resistant to apoptosis, what's more, the intensity of global apoptosis and autophagy clearance significantly declines in humans during aging, as aging repress the apoptotic response. In conclusion, apoptosis is the reasonable result of the aging process, and aging enhance the cells and organisms apoptosis, however, aging process resist apoptosis as a crucial defense mechanism against sublethal damage. On the other hand, apoptosis is benefit to aging and aging process, as it is important to organ to maintain homeostasis and protect against age-associated tumorigenesis. Numerous studies have indicated that anticancer therapies primarily act by activating the apoptosis response pathway in tumor cells, so apoptosis may be used in exploring the mechanisms of tumorigenesis and anticancer therapy.

\section{REFERENCES}

[1] Kerr, J.F., Wyllie, A.H. and Currie, A.R. (1972) Apoptosis: A basic biological phenomenon with wide-ranging implications in tissue kinetics. British Journal of Cancer, 4, 239-257. doi:10.1038/bjc.1972.33

[2] Verheij, M. and Bartelink, H. (2000) Radiation-induced apoptosis. Cell and Tissue Research, 1, 133-142. doi:10.1007/s004410000188

[3] Chen, W., et al. (2008) p53-related apoptosis resistance and tumor suppression activity in UVB-induced premature senescent human skin fibroblasts. International Journal of Molecular Medicine, 5, 645-653.

[4] Lettre, G. and Hengartner, M.O. (2006) Developmental apoptosis in C. elegans: A complex CEDnario. Nature Reviews Molecular Cell Biology, 2, 97-108. doi:10.1038/nrm1836

[5] Pecina-Slaus, N. (2010) Wnt signal transduction pathway and apoptosis: A review. Cancer Cell International, 10, 22. doi:10.1186/1475-2867-10-22

[6] Oberdoerffer, P. and Sinclair, D.A. (2007) The role of nuclear architecture in genomic instability and ageing. Nature Reviews Molecular Cell Biology, 9, 692-702. doi:10.1038/nrm2238

[7] Fischer, U., Janicke, R.U. and Schulze-Osthoff, K. (2003) Many cuts to ruin: A comprehensive update of caspase substrates. Cell Death \& Differentiation, 1, 76-100. doi:10.1038/sj.cdd.4401160

[8] Chipuk, J.E. and Green, D.R. (2008) How do BCL-2 proteins induce mitochondrial outer membrane permeabilization? Trends in Cell Biology, 4, 157-164. doi:10.1016/j.tcb.2008.01.007

[9] Griner, E.M. and Kazanietz, M.G. (2007) Protein kinase $\mathrm{C}$ and other diacylglycerol effectors in cancer. Nature Reviews Cancer, 4, 281-294. doi:10.1038/nrc2110

[10] Ramakrishna, S., Suresh, B. and Baek, K.H. (2011) The role of deubiquitinating enzymes in apoptosis. Cellular and Molecular Life Sciences, 1, 15-26. doi:10.1007/s00018-010-0504-6

[11] Kurz, T., Terman, A., Gustafsson, B. and Brunk, U.T. (2008) Lysosomes in iron metabolism, ageing and apoptosis. Histochemistry and Cell Biology, 4, 389-406. doi:10.1007/s00418-008-0394-y

[12] Estaquier, J., et al. (2012) The mitochondrial pathways of apoptosis. Advances in Experimental Medicine and Biology, 157-83. doi:10.1007/978-94-007-2869-1_7

[13] Freund, A., et al. (2010) Inflammatory networks during cellular senescence: Causes and consequences. Trends in Molecular Medicine, 5, 238-246. doi:10.1016/j.molmed.2010.03.003

[14] Circu, M.L. and Aw, T.Y. (2010) Reactive oxygen species, cellular redox systems, and apoptosis. Free Radical 
Biology \& Medicine, 6, 749-762. doi:10.1016/j.freeradbiomed.2009.12.022

[15] Maiuri, M.C., et al. (2007) Self-eating and self-killing: Crosstalk between autophagy and apoptosis. Nature Reviews Molecular Cell Biology, 9, 741-752. doi:10.1038/nrm2239

[16] Harman, D. (2001) Aging: Overview. Annals of the New York Academy of Sciences, 928, 1-21. doi:10.1111/j.1749-6632.2001.tb05631.x

[17] Rattan, S.I. (2006) Theories of biological aging: Genes, proteins, and free radicals. Free Radical Research, 12, 1230-1238. doi:10.1080/10715760600911303

[18] Muller, M. (2009) Cellular senescence: Molecular mechanisms, in vivo significance, and redox considerations. Antioxidants \& Redox Signaling, 1, 59-98. doi:10.1089/ars.2008.2104

[19] Weinert, B.T. and Timiras, P.S. (2003) Invited review: Theories of aging. Journal of Applied Physiology, 4, 1706-1716.

[20] Troen, B.R. (2003) The biology of aging. Mount Sinai Journal of Medicine, 1, 3-22.

[21] Salminen, A. and Kaarniranta, K. (2010) Genetics vs. entropy: Longevity factors suppress the NF-kappaBdriven entropic aging process. Ageing Research Reviews, 3, 298-314. doi:10.1016/j.arr.2009.11.001

[22] Warner, H.R. (1997) Aging and regulation of apoptosis. Current Topics in Cellular Regulation, 35, 107-121. doi:10.1016/S0070-2137(97)80004-0

[23] Warner, H.R. (1999) Apoptosis: A two-edged sword in aging. Annals of the New York Academy of Sciences, 887, 1-11. doi:10.1111/j.1749-6632.1999.tb07917.x

[24] Higami, Y. and Shimokawa, I. (2000) Apoptosis in the aging process. Cell and Tissue Research, 1, 125-132. doi:10.1007/s004419900156

[25] Muradian, K. and Schachtschabel, D.O. (2001) The role of apoptosis in aging and age-related disease: Update. Zeitschrift für Gerontologie und Geriatrie, 6, 441-446. doi:10.1007/s003910170015

[26] Alt, E.U., et al. (2012) Aging alters tissue resident mesenchymal stem cell properties. Stem Cell Research, 2, 215-225. doi:10.1016/j.scr.2011.11.002

[27] Wilson, A., et al. (2010) Age-related molecular genetic changes of murine bone marrow mesenchymal stem cells. BMC Genomics, 11, 229. doi:10.1186/1471-2164-11-229

[28] Todd, A.R., et al. (2004) Characterization of mature vs aged rabbit articular cartilage: Analysis of cell density, apoptosis-related gene expression and mechanisms controlling chondrocyte apoptosis. Osteoarthritis and Cartilage, 11, 917-923.

[29] Cao, J.N., et al. (2010) Age-related alterations of gene expression patterns in human CD8+ T cells. Aging Cell, 1, 19-31. doi:10.1111/j.1474-9726.2009.00534.x

[30] Tyner, S.D, et al. (2002) p53 mutant mice that display early ageing-associated phenotypes. Nature, 6867, 45-53. doi:10.1038/415045a

[31] Maier, B., et al. (2004) Modulation of mammalian life span by the short isoform of p53. Genes \& Development,

\section{3, 306-319. doi:10.1101/gad.1162404}

[32] Tsujimoto, Y. (2003) Cell death regulation by the Bcl-2 protein family in the mitochondria. Journal of Cellular Physiology, 2, 158-167. doi:10.1002/jcp.10254

[33] Kujoth, G.C., et al. (2005) Mitochondrial DNA mutations, oxidative stress, and apoptosis in mammalian aging. Science, 5733, 481-484. doi:10.1126/science.1112125

[34] Gupta, S. (2005) Molecular mechanisms of apoptosis in the cells of the immune system in human aging. Immunological Reviews, 205, 114-129. doi:10.1111/j.0105-2896.2005.00261.x

[35] Johnstone, R.W., et al. (2002) Apoptosis: A link between cancer genetics and chemotherapy. Cell, 2, 153-164. doi:10.1016/S0092-8674(02)00625-6

[36] Su, J.H., et al. (2002) Caspase-cleaved amyloid precursor protein and activated caspase- 3 are co-localized in the granules of granulovacuolar degeneration in Alzheimer's disease and Down's syndrome brain. Acta Neuropathologica, 1, 1-6. doi:10.1007/s00401-002-0548-2

[37] Bredesen, D.E., et al. (2006) Cell death in the nervous system. Nature, 7113, 796-802. doi:10.1038/nature05293

[38] Mattson, M.P. (2000) Apoptosis in neurodegenerative disorders. Nature Reviews Molecular Cell Biology, 2, 120-129. doi:10.1038/35040009

[39] Soo, K.Y., et al. (2012) Bim links ER stress and apoptosis in cells expressing mutant SOD1 associated with amyotrophic lateral sclerosis. PLoS One, 4, e35413. doi:10.1371/journal.pone.0035413

[40] Milnerwood, A.J., et al. (2012) Mitigation of augmented extrasynaptic NMDAR signaling and apoptosis in cortico-striatal co-cultures from Huntington's disease mice. Neurobiology of Disease, 1, 40-51. doi:10.1016/j.nbd.2012.05.013

[41] Pollack, M., et al. (2002) The role of apoptosis in the normal aging brain, skeletal muscle, and heart. Annals of the New York Academy of Sciences, 959, 93-107. doi:10.1111/j.1749-6632.2002.tb02086.x

[42] Jejurikar, S.S., et al. (2006) Aging increases the susceptibility of skeletal muscle derived satellite cells to apoptosis. Experimental Gerontology, 9, 828-836. doi:10.1016/j.exger.2006.06.053

[43] Zhang, Y. and Herman, B. (2002) Ageing and apoptosis. Mechanisms of Ageing and Development, 4, 245-260. doi:10.1016/S0047-6374(01)00349-9

[44] Proskuryakov, S.Y., et al. (2005) Immunology of apoptosis and necrosis. Biochemistry, 12, 1310-1320.

[45] Suh, Y. (2002) Cell signaling in aging and apoptosis. Mechanisms of Ageing and Development, 8, 881-890. doi:10.1016/S0047-6374(02)00025-8

[46] Zhang, W., et al. (2005) UVB-induced apoptosis drives clonal expansion during skin tumor development. Carcinogenesis, 1, 249-257.

[47] Rochette, P.J. and Brash, D.E. (2008) Progressive apoptosis resistance prior to senescence and control by the anti-apoptotic protein BCL-xL. Mechanisms of Ageing and Development, 4, 207-214. doi:10.1016/j.mad.2007.12.007 
[48] Rai, N.K., et al. (2005) Apoptosis: A basic physiologic process in wound healing. The International Journal of Lower Extremity Wounds, 3, 138-144. doi:10.1177/1534734605280018

[49] Gosain, A. and DiPietro, L.A. (2004) Aging and wound healing. World Journal of Surgery, 3, 321-326. doi:10.1007/s00268-003-7397-6

[50] Ballas, C.B. and Davidson, J.M. (2001) Delayed wound healing in aged rats is associated with increased collagen gel remodeling and contraction by skin fibroblasts, not with differences in apoptotic or myofibroblast cell populations. Wound Repair and Regeneration, 3, 223-237. doi:10.1046/j.1524-475x.2001.00223.x

[51] DePinho, R.A. (2000) The age of cancer. Nature, 6809, 248-254. doi:10.1038/35041694

[52] Camplejohn, R.S., et al. (2003) Apoptosis, ageing and cancer susceptibility. British Journal of Cancer, 4, 487490. doi:10.1038/sj.bjc.6600767

[53] Suh, Y., et al. (2002) Aging alters the apoptotic response to genotoxic stress. Nature Medicine, 1, 3-4.

[54] Polyak, K., et al. (1997) Less death in the dying. Cell Death \& Differentiation, 3, 242-246. doi:10.1038/sj.cdd.4400226

[55] Bassi, P. and Sacco, E. (2009) Cancer and aging: The molecular pathways. Urologic Oncology, 6, 620-627. doi:10.1016/j.urolonc.2009.07.013

[56] Feng, Z., et al. (2008) The tumor suppressor p53: Cancer and aging. Cell Cycle, 7, 842-847. doi:10.4161/cc.7.7.5657

[57] Vallon, M., et al. (2012) Enhanced efficacy of combined (213) Bi-DTPA-F3 and paclitaxel therapy of peritoneal carcinomatosis is mediated by enhanced induction of apoptosis and G2/M phase arrest. European Journal of Nuclear Medicine and Molecular Imaging, in Press. doi:10.1007/s00259-012-2203-z

[58] Salom, E., et al. (2012) Correlation of pretreatment drug induced apoptosis in ovarian cancer cells with patient survival and clinical response. Journal of Translational Medicine, 1, 162. doi:10.1186/1479-5876-10-162

[59] Bosserman, L., et al. (2012) The microculture-kinetic (MiCK) assay: The role of a drug-induced apoptosis assay in drug development and clinical care. Cancer Research, 72, 3901-3905. doi:10.1158/0008-5472.CAN-12-0681

[60] Herr, I. and Debatin, K.M. (2001) Cellular stress response and apoptosis in cancer therapy. Blood, 9, 2603-2614. doi:10.1182/blood.V98.9.2603

[61] Kaufmann, S.H. and Earnshaw, W.C. (2000) Induction of apoptosis by cancer chemotherapy. Experimental Cell Research, 1, 42-49. doi:10.1006/excr.2000.4838

[62] Hornsby, P.J. (2010) Senescence and life span. Pflügers Archiv, 2, 291-299. doi:10.1007/s00424-009-0723-6

[63] Chondrogianni, N., et al. (2003) Central role of the proteasome in senescence and survival of human fibroblasts: Induction of a senescence-like phenotype upon its inhibition and resistance to stress upon its activation. The Journal of Biological Chemistry, 30, 28026. doi:10.1074/jbc.M301048200
[64] Spillare, E.P., et al. (1999) p53-mediated apoptosis is attenuated in Werner syndrome cells. Genes \& Development, 11, 1355-1360. doi:10.1101/gad.13.11.1355

[65] Campisi, J., et al. (2007) Cellular senescence: When bad things happen to good cells. Nature Reviews Molecular Cell Biology, 9, 729-740. doi:10.1038/nrm2233

[66] Marcotte, R., et al. (2004) Senescent fibroblasts resist apoptosis by downregulating caspase-3. Mechanisms of Ageing and Development, 10-11, 777-783. doi:10.1016/j.mad.2004.07.007

[67] Jackson, J.K., et al. (2006) p53 is preferentially recruited to the promoters of growth arrest genes p21 and GADD45 during replicative senescence of normal human fibroblasts. Cancer Research, 17, 8356-8360. doi:10.1158/0008-5472.CAN-06-1752

[68] Chaturvedi, V., et al. (2004) Resistance to UV-induced apoptosis in human keratinocytes during accelerated senescence is associated with functional inactivation of p53. Journal of Cellular Physiology, 1, 100-109. doi:10.1002/jcp.10392

[69] Hampel, B., et al. (2004) Differential regulation of apoptotic cell death in senescent human cells. Experimental Gerontology, 11-12, 1713-1721. doi:10.1016/j.exger.2004.05.010

[70] Labinskyy, N., et al. (2006) Comparison of endothelial function, $\mathrm{O}_{2}$-* $^{*}$ and $\mathrm{H}_{2} \mathrm{O}_{2}$ production, and vascular oxidative stress resistance between the longest-living rodent, the naked mole rat, and mice. American Journal of Physiology-Heart and Circulatory Physiology, 6, H2698704. doi:10.1152/ajpheart.00534.2006

[71] Chiarugi, P. and Giannoni, E. (2008) Anoikis: A necessary death program for anchorage-dependent cells. Biochemical Pharmacology, 11, 1352-1364. doi:10.1016/j.bcp.2008.07.023

[72] Kavathia, N., et al. (2009) Serum markers of apoptosis decrease with age and cancer stage. Aging, 7, 652-663.

[73] Hinkal, G.W., et al. (2009) Altered senescence, apoptosis, and DNA damage response in a mutant p53 model of accelerated aging. Mechanisms of Ageing and Development, 4, 262-271. doi:10.1016/j.mad.2009.01.001

[74] Devitt, A. and Marshall, L.J. (2011) The innate immune system and the clearance of apoptotic cells. Journal of Leukocyte Biology, 3, 447-457. doi:10.1189/jlb.0211095

[75] De Martinis, M., et al. (2007) Apoptosis remodeling in immunosenescence: Implications for strategies to delay ageing. Current Medicinal Chemistry, 13, 1389-1397. doi:10.2174/092986707780831122

[76] Rajawat, Y.S., et al. (2009) Aging: Central role for autophagy and the lysosomal degradative system. Ageing Research Reviews, 3, 199-213. doi:10.1016/j.arr.2009.05.001

[77] Salminen, A. and Kaarniranta, K. (2009) Regulation of the aging process by autophagy. Trends in Molecular Medicine, 5, 217-224. doi:10.1016/j.molmed.2009.03.004

[78] Young, A.R. and Narita, M. (2010) Connecting autophagy to senescence in pathophysiology. Current Opinion in Cell Biology, 2, 234-240. doi:10.1016/j.ceb.2009.12.005 
[79] Young, A.R., et al. (2009) Autophagy mediates the mitotic senescence transition. Genes \& Development, 7, 798-803. doi:10.1101/gad.519709

[80] Marino, G., et al. (2008) Premature aging in mice activates a systemic metabolic response involving autophagy induction. Human Molecular Genetics, 14, 2196-2211. doi:10.1093/hmg/ddn120

[81] Yang, K., et al. (2010) Advanced glycation end products induce chemokine/cytokine production via activation of p38 pathway and inhibit proliferation and migration of bone marrow mesenchymal stem cells. Cardiovascular Diabetology, 9, 66. doi:10.1186/1475-2840-9-66 